\title{
Crecimiento y rendimiento comercial de Pinus elliottii en plantación y en regeneración natural manejada con raleos en Entre Ríos, Argentina
}

\author{
Growth and commercial yield of Pinus elliottii in planting and natural regeneration \\ managed with thinnings in Entre Ríos, Argentina
}

\author{
Silvia L Rebottaro**, Daniel A Cabrellia \\ *Autor de correspondencia: anniversidad de Buenos Aires, Facultad de Agronomía, Cátedra de Dasonomía, \\ Av. San Martín 4453 (1417), Buenos Aires, Argentina, Tel.: 54-11-45248091, rebottar@agro.uba.ar
}

\begin{abstract}
SUMMARY
Pinus elliottii is an exotic species very much cultivated in Argentina, with potential to regenerate naturally. When high density ocurres, a control over initial stocking is necessary. The object of the work was to evaluate the effects of the early thinning of $P$. elliottii natural regeneration and its behavior concerning planting during six years. Treatments were: natural regeneration without thinning with 17,000 trees ha ${ }^{-1}(\mathrm{SR})$, thinning with residual density of 5,000 trees ha-1 $\left(\mathrm{R}_{1}\right)$, thinning with density of 2,500 trees ha $^{-1}\left(\mathrm{R}_{2}\right)$, and planting with 800 trees ha- $\mathrm{a}^{-1}(\mathrm{P})$. Diameter and height growth, allometric relationships, cylindrical form factor, size of the branch, basal area (AB), density index (IDR), and commercial yield were evaluated. Results indicated that natural regeneration has better initial performance contrasted to P. Early thinning of natural regeneration has positive effect on diameter and height growth. Trees in SR had most cylindrical form; whereas in P, trees had most conical form. Branches diameter and length decrease as density of trees increases. After six years of treatments, SR reached highest AB and volume ha ${ }^{-1}$, but lacking commercial value. Independently from intensity of thinning applied, early control of density produced high wood volume although presenting small sizes (diameter $\geq 7 \mathrm{~cm}$ ). The $R_{2}$ treatment reached the highest commercial wood volume presenting diameters $\geq 10 \mathrm{~cm}$. In the context of density management of the species, the results obtained of IDR and individual growth were discussed.
\end{abstract}

Key words: Pinus elliottii, natural regeneration, thinning, growth, commercial yield.

RESUMEN

Pinus elliottii es una especie exótica muy cultivada en Argentina, con alto potencial para regenerar naturalmente. Cuando la densidad de la regeneración es elevada se requiere un manejo temprano de la misma. El objetivo de este estudio fue evaluar el efecto del raleo temprano de la regeneración de P. elliottii y su comportamiento con relación a una plantación, durante seis años. Los tratamientos fueron: regeneración sin raleo con 17.000 árboles ha ${ }^{-1}(\mathrm{SR})$, raleo dejando 5.000 árboles ha ${ }^{-1}\left(\mathrm{R}_{1}\right)$, raleo dejando 2.500 árboles ha $\mathrm{ha}^{-1}\left(\mathrm{R}_{2}\right)$ y una plantación con 800 árboles ha $\mathrm{h}^{-1}(\mathrm{P})$. Las variables evaluadas fueron: crecimiento en diámetro y altura, alometría de las plantas, coeficiente de forma, tamaño de ramas, área basal (AB), índice de densidad (IDR) y rendimiento comercial. Los resultados indicaron que la regeneración natural tuvo mejor comportamiento inicial que $\mathrm{P}$. El raleo temprano tuvo un efecto positivo sobre el crecimiento en diámetro y altura. En SR las plantas fueron más cilíndricas, mientras que en $\mathrm{P}$ resultaron más cónicas. El diámetro y longitud de las ramas disminuyó con el aumento de la densidad. Después de seis años de tratamiento, SR alcanzó mayor $\mathrm{AB}$ y volumen $\mathrm{ha}^{-1}$, pero sin valor comercial. En cambio, independientemente de la intensidad del raleo, el control de la densidad generó mucho volumen de madera de pequeñas dimensiones (diámetro $\geq 7 \mathrm{~cm}$ ). Pero el mayor volumen de madera con diámetro $\geq 10 \mathrm{~cm}$, se logró en $\mathrm{R}_{2}$. Los datos de IDR y crecimiento individual fueron discutidos en el contexto del manejo de la densidad de la especie.

Palabras clave: Pinus elliottii, regeneración natural, raleo, crecimiento, rendimiento comercial.

\section{INTRODUCCIÓN}

Pinus elliottii (Engelm.) es una de las especies forestales más cultivadas en Argentina, siendo su madera utilizada para el aserrado, la industria papelera y la producción de tableros (SAGyP 1999). Esta especie, originaria del sudeste de Estados Unidos, se ha adaptado exitosamente a un amplio rango de condiciones ecológicas en nuestro país, alcanzando elevados niveles de productividad. En diferentes zonas de cultivo es frecuente encontrar rodales establecidos a partir de regeneración natural con diferente grado de desarrollo (Lombardi 1982, De Bellis 1986, 
Brassiolo y Seitz 1988, Cozzo et al. 1988, Torres et al. 1994). Dicho proceso ha alcanzado su máxima expresión en la Mesopotamia Argentina (al noreste del país), pudiendo constituir un recurso atractivo tanto desde el punto de vista económico como ecológico (Cozzo et al. 1988, Cabrelli et al. 1997, Lombardi 1982).

La mayoría de los estudios sobre el establecimiento y manejo de la regeneración natural de $P$. elliottii proviene principalmente del área de origen de la especie (McCulley 1950, Langdon y Bennett 1976, Backer y Muphy 1982, Cain 1987, Edwards 1987, Lohrey y Kossuth 1990, Cain 1991, Dangerfield y Edwards 1991, Cain 1996, Cain y Shelton 2001). Es conocido que en muchas especies de pino la semilla de los años anteriores tiene poco valor regenerativo, y que el éxito de la regeneración depende principalmente de la semilla diseminada durante el año en curso (Barnett y McGilvray 1991). Resultados similares fueron obtenidos en Argentina para P. elliottii (Méndez 1996). Según Langdon (1981) se requieren al menos 75.000 semillas ha $^{-1}$ en el suelo para que el establecimiento de la regeneración natural después de una tala rasa sea exitoso. En años con abundante producción de semilla y con condiciones climáticas favorables (principalmente de humedad), la densidad inicial de plantas puede llegar a ser muy alta. En Argentina se han registrado rodales con una densidad de 160.000 árboles ha ${ }^{-1}$ dentro del primer año posterior a la tala rasa (Cabrelli et al. 1997).

El uso de la regeneración natural es una alternativa productiva que tiene ventajas y desventajas. Algunas de las ventajas son el bajo costo inicial, las menores labores durante el establecimiento y el menor impacto sobre el suelo. Entre las desventajas se menciona principalmente la necesidad de realizar un control temprano de la densidad, lo que obliga a realizar un raleo precomercial (Edwards 1987). Otra desventaja se asocia con el hecho de utilizar un material genético de menor calidad respecto al usado en las plantaciones nuevas. Sin embargo, cuando la densidad de plantas es muy alta, existe una fuerte presión de selección ejercida en el raleo precomercial, y ello garantiza en parte que el ciclo productivo se inicie con muy buenos individuos. En algunos casos, incluso, se ha registrado un mejor comportamiento inicial de la regeneración natural respecto a la plantación (Ackzell 1993). Debido a que P. elliottii es una especie heliófita, es necesario generar aperturas grandes para favorecer el desarrollo inicial de la regeneración (Edwards 1987). Luego del establecimiento, esa característica ecofisiológica influye sobre el alto potencial de respuesta al raleo de la especie. Duryea (1987) reporta que existe una fuerte restricción en el crecimiento de la regeneración natural de $P$. elliottii cuando la densidad a los tres años de edad supera los 5.000 árboles ha ${ }^{-1}$. Mann y Lohrey (1974) recomiendan efectuar un raleo precomercial cuando la densidad excede los 12.500 árboles $\mathrm{ha}^{-1}$.

En Argentina, los valores de crecimiento inicial de plantas de $P$. elliottii regeneradas naturalmente resultan promisorios
(Cabrelli et al. 1997, Rebottaro et al. 2001, 2002, 2004). Pero no siempre un buen crecimiento es equivalente a un buen rendimiento comercial, ya que este último depende de la densidad de plantas, de la estructura de tamaños de la población y de los estándares de comercialización de cada región. En este sentido se debería saber si la reducción drástica de la densidad en rodales regenerados naturalmente producirá una respuesta significativa sobre el crecimiento en términos comerciales.

En este marco el presente trabajo tiene como objetivo evaluar el efecto de dos intensidades de raleo temprano de la regeneración natural de $P$. elliottii, y su comportamiento con relación a una plantación tradicional, durante seis años.

\section{MÉTODOS}

Descripción del sitio experimental. El trabajo de campo se llevó a cabo en la Estación Experimental Yuquerí dependiente del Instituto Nacional de Tecnología Agropecuaria (INTA), ubicada en Concordia ( $31^{\circ} 22^{\prime}$ Lat. S, $58^{\circ} 07^{\prime}$ Long. O, 47 m s.n.m.), provincia de Entre Ríos, Argentina. El clima en la región es templado a subtropical húmedo, con una temperatura media anual de $18,5^{\circ} \mathrm{C}$. La temperatura media del mes más frío (julio) es de $12{ }^{\circ} \mathrm{C}$ y la media del mes más cálido (enero) es de $25^{\circ} \mathrm{C}$. La precipitación media anual es de $1.274 \mathrm{~mm}$, sin estacionalidad marcada, aunque generalmente ocurre déficit hídrico a fines de primavera-principios de verano (SMN 1992). El trabajo experimental se llevó a cabo en una zona con suelo arenoso pardo (Entisoles), con profundidad efectiva mayor a $60 \mathrm{~cm}$, con baja capacidad de retención hídrica y bajo contenido de materia orgánica y nutrientes (Rebottaro y Cabrelli 2004).

Instalación del ensayo y tratamientos. En el año 1994 una plantación de 3 ha de $P$. elliottii de 24 años de edad fue sometida a una tala rasa. Unos meses después el lote fue ocupado por regeneración natural de la especie con una densidad de 160.000 plantas ha $^{-1}$ (Cabrelli et al. 1997). En junio del año 1996, sobre este lote fue instalado un experimento de raleo precomercial. Los tratamientos aplicados fueron: regeneración natural sin raleo con una densidad de 17.000 árboles $\mathrm{ha}^{-1}(\mathrm{SR})$, raleo con una densidad residual de 5.000 árboles $\mathrm{ha}^{-1}\left(\mathrm{R}_{1}\right)$ y raleo dejando 2.500 árboles $\mathrm{ha}^{-1}\left(\mathrm{R}_{2}\right)$. Para cada tratamiento se realizaron cuatro repeticiones (parcelas de 30x50 m), asignando los tratamientos en forma aleatoria sobre un total de 12 parcelas. El raleo se realizó por lo bajo y en forma manual. En cada parcela se identificaron 20 plantas, usando chapas de aluminio numeradas. Con el fin de evitar el efecto de borde, las plantas utilizadas para el estudio fueron las ubicadas en el centro de cada parcela. En el tratamiento sin raleo, el criterio utilizado para la elección de los árboles a marcar, fue que éstos tuvieran un tamaño similar a las plantas 
de los tratamientos con raleo. Como el raleo se hizo por lo bajo, los árboles marcados en el tratamiento sin raleo fueron los de mayor tamaño.

Con el fin de comparar la regeneración natural, se evaluó una plantación $(\mathrm{P})$ de $P$. elliottii de 2 ha, establecida en abril de 1995. Dicha plantación se encontraba separada de la regeneración por una calle forestal de $8 \mathrm{~m}$ de ancho. La plantación era una reforestación de pino sobre pino, con un distancimiento inicial de $3,5 \times 3,5 \mathrm{~m}$, y plantas producidas en el vivero del INTA a partir de semillas del huerto semillero INTA-Cerro Azul (Misiones, Argentina). En dicha plantación se instalaron cuatro parcelas donde se marcaron 20 árboles en cada una. Los individuos elegidos fueron aquellos que habían tenido un buen establecimiento (evaluado en términos de tamaño, vigor y sanidad). No fueron considerados los sitios de plantación cuyos individuos provenían de una reposición de falla.

Mediciones. Con el objeto de realizar mediciones del diámetro en la base del tallo (DBT) los árboles seleccionados fueron marcados con pintura a $10 \mathrm{~cm}$ del nivel del suelo. El DBT y la altura total (A) de todas las plantas marcadas fueron medidos en junio de 1996, 1997, 1998 y 2002. El diámetro a la altura del pecho (DAP) fue marcado y medido en junio de 1997, 1998, 1999, 2000, 2001 y 2002. Los datos del área transversal promedio (ATP) y del número árboles ha ${ }^{-1}$ fueron utilizados para calcular el área basal (AB). Con la finalidad de evaluar la evolución de la densidad relativa, el diámetro cuadrático medio (diámetro del ATP) y el número de árboles $\mathrm{ha}^{-1}$ fueron utilizados para calcular el Índice de Densidad del Rodal (IDR) de Reineke (1933).

En febrero de 2002 se seleccionaron cuatro plantas por tratamiento (una por repetición), y se efectuó un análisis destructivo para determinar el Coeficiente de Forma (CF). Los individuos elegidos correspondieron a árboles de ATP. En esos individuos se midió DBT, DAP, altura y los diámetros a intervalos de un metro desde el DAP hacia el ápice. El sector apical del árbol $(1 \mathrm{~m}<\operatorname{largo}<2 \mathrm{~m})$ fue considerado de forma cónica.

Usando los datos de DAP, altura y número árboles por parcela del inventario del año 2002, y el CF obtenido de cada repetición, se calculó el volumen total de madera por parcela, y se extrapoló su valor a volumen total de madera por ha (VT). También se calculó el volumen comercial con diámetro en punta fina $\geq 7 \mathrm{~cm}\left(\mathrm{VC}_{\geq 7}\right)$, y diámetro $\geq 10 \mathrm{~cm}\left(\mathrm{VC}_{\geq 10}\right)$. En ambos casos el largo comercial de trozas fue $\geq 2 \mathrm{~m}$. Para el cálculo del $\mathrm{VC}_{\geq 7} \mathrm{y} \mathrm{VC}_{\geq 10}$ se utilizó el mismo inventario que para el cálculo del VT, pero aplicando en la función de volumen un coeficiente de forma comercial. Dicho coeficiente fue estimado a partir de los mismos árboles usados para el cálculo de $\mathrm{CF}$, pero considerando solamente el volumen de madera comprendida en el árbol bajo las dos restricciones de tamaño mencionadas anteriormente.
Los mismos árboles utilizados para calcular el CF fueron usados para evaluar la dimensión de las ramas. Las poblaciones utilizadas en este caso fueron SR, P y el tratamiento de raleo más intenso $\left(\mathrm{R}_{2}\right)$. Se midió el diámetro basal (Dr) y la longitud ( $\mathrm{Lr}$ ) de todas las ramas en cada árbol seleccionado.

En todos los casos los diámetros fueron medidos con calibre digital (con precisión de $0,01 \mathrm{~mm}$ ) y la altura con vara telescópica (con precisión de $0,1 \mathrm{~cm}$ ). La longitud de las ramas se midió con cinta métrica (con precisión de $0,1 \mathrm{~cm})$.

Análisis de los datos. Las poblaciones fueron caracterizadas al inicio del estudio a través de la A, el DBT y la relación A/DBT. Se analizó el crecimiento en diámetro (DBT y DAP) y altura, las relaciones alométricas, el CF y el tamaño de las ramas después de seis años. El efecto de los tratamientos se evaluó mediante análisis de varianza y la posterior comparación de medias a través de la prueba de Tukey. Se consideraron valores de significancia con $P<0,05$. A los fines de evaluar el efecto de la densidad sobre el crecimiento individual a través del tiempo, se utilizaron los datos del DAP para calcular el incremento corriente anual (ICA) y el incremento medio anual (IMA) de cada tratamiento. También se calcularon la evolución del AB y del IDR como índice de competencia, y los valores de volumen total y comercial al final del estudio. Los análisis estadísticos fueron realizados con el programa para análisis estadístico SAS (2001).

\section{RESULTADOS}

Tamaño inicial de las plantas. Al comienzo del estudio no se registraron diferencias significativas en el tamaño de las plantas entre las tres densidades de regeneración natural (cuadro 1). Esto debido a que para evaluar el tratamiento SR se eligieron plantas que tenían tamaño inicial parecido al de los árboles residuales de los tratamientos con raleo. En cambio, se obtuvieron diferencias significativas, aunque de pequeña magnitud, entre la regeneración natural y la plantación. La menor relación altura/diámetro en P mostró que, desde el punto de vista morfológico, las plantas fueron más bajas con relación a su diámetro comparado con las regeneradas naturalmente.

Crecimiento individual. La evaluación del incremento del DBT durante los dos primeros años del estudio indicó que la tendencia respecto a un mejor comportamiento inicial de la regeneración natural, se mantuvo durante la segunda estación de crecimiento (figura 1). Recién a partir del tercer año la plantación comenzó a tener un mayor crecimiento, aunque no se diferenció significativamente del tratamiento con raleo más intenso $\left(\mathrm{R}_{2}\right)$.

La evolución del ICA y del IMA en diámetro a través del tiempo permite visualizar el efecto de la competencia sobre el crecimiento (figura 2). En este sentido, podemos 
tomar a la plantación como una situación donde no hubo competencia por varios años, y en el otro extremo se ubica la regeneración natural sin raleo, donde la alta densidad inicial generó altos niveles de competencia tempranamente.

Cuadro 1. Media ( \pm desviación estándar) de la altura total (A), el diámetro a la base del tallo (DBT), y la relación A/DBT en plantas de Pinus elliottii al inicio del ensayo, para cada tratamiento: regeneración natural sin raleo (SR), raleo dejando 5.000 árboles ha $^{-1}\left(\mathrm{R}_{1}\right)$; raleo dejando 2.500 árboles ha ${ }^{-1}\left(\mathrm{R}_{2}\right)$ y plantación con 800 árboles ha ${ }^{-1}(\mathrm{P})$. Las letras diferentes en cada columna indican diferencias entre tratamientos (Tukey, $P<0,05$ ).

Mean ( \pm standard deviation) of total height (A), basal diameter (DBT), and total height/basal diameter (A/DBT) in Pinus elliottii seedlings, at the beginning of the study for each treatment: natural regeneration without thinning (SR), thinning to residual density of 5,000 seedlings $\mathrm{ha}^{-1}\left(\mathrm{R}_{1}\right)$, thinning to residual density of 2,500 seedlings ha $\mathrm{h}^{-1}\left(\mathrm{R}_{2}\right)$, and planting with 800 seedlings ha ${ }^{-1}(\mathrm{P})$. Different letters in each column designate significant differences among treatments (Tukey, $P<0.05$ ).

\begin{tabular}{lrrc}
\hline & A $(\mathrm{cm})$ & DBT $(\mathrm{mm})$ & A/DBT $(\mathrm{cm} / \mathrm{mm})$ \\
\hline $\mathrm{SR}$ & $143,2 \pm 26,6 \mathrm{a}$ & $30,70 \pm 6,84 \mathrm{a}$ & $4,78 \pm 1,02 \mathrm{a}$ \\
$\mathrm{R}_{1}$ & $144,0 \pm 17,2 \mathrm{a}$ & $29,50 \pm 5,80 \mathrm{a}$ & $4,64 \pm 0,57 \mathrm{a}$ \\
$\mathrm{R}_{2}$ & $141,5 \pm 18,4 \mathrm{a}$ & $30,17 \pm 6,37 \mathrm{a}$ & $4,84 \pm 1,05 \mathrm{a}$ \\
$\mathrm{P}$ & $80,0 \pm 19,2 \mathrm{~b}$ & $26,30 \pm 7,38 \mathrm{~b}$ & $3,13 \pm 0,65 \mathrm{~b}$ \\
\hline
\end{tabular}

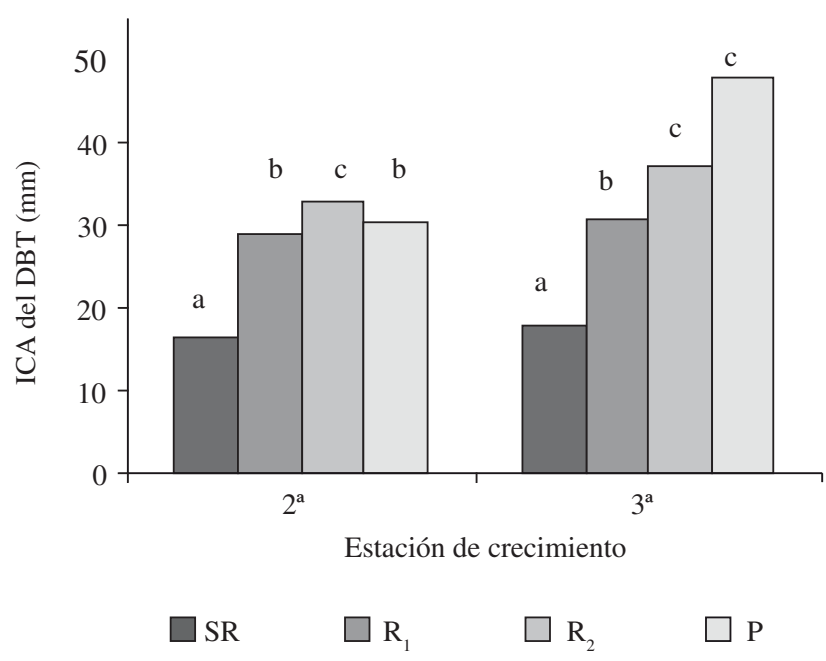

Figura 1. Incremento corriente anual del diámetro en la base del tallo (DBT) en plantas de Pinus elliottii, para cada tratamiento: regeneración natural sin raleo (SR), raleo dejando 5.000 árboles $\mathrm{ha}^{-1}\left(\mathrm{R}_{1}\right)$, raleo dejando 2.500 árboles ha ${ }^{-1}\left(\mathrm{R}_{2}\right)$ y plantación con 800 árboles $\mathrm{ha}^{-1}(\mathrm{P})$. Las letras diferentes indican diferencias entre tratamientos (Tukey, $P<0,05$ ).

Current annual increment of the basal diameter (DBT), in Pinus elliottii seedlings for each treatment: natural regeneration without thinning (SR), thinning to residual density of 5,000 seedlings ha ${ }^{-1}\left(\mathrm{R}_{1}\right)$, thinning to residual density of 2,500 seedlings $\mathrm{ha}^{-1}\left(\mathrm{R}_{2}\right)$, and planting with 800 seedlings ha ${ }^{-1}(\mathrm{P})$. Different letters designate significant differences among treatments (Tukey, $P<0.05$ ).
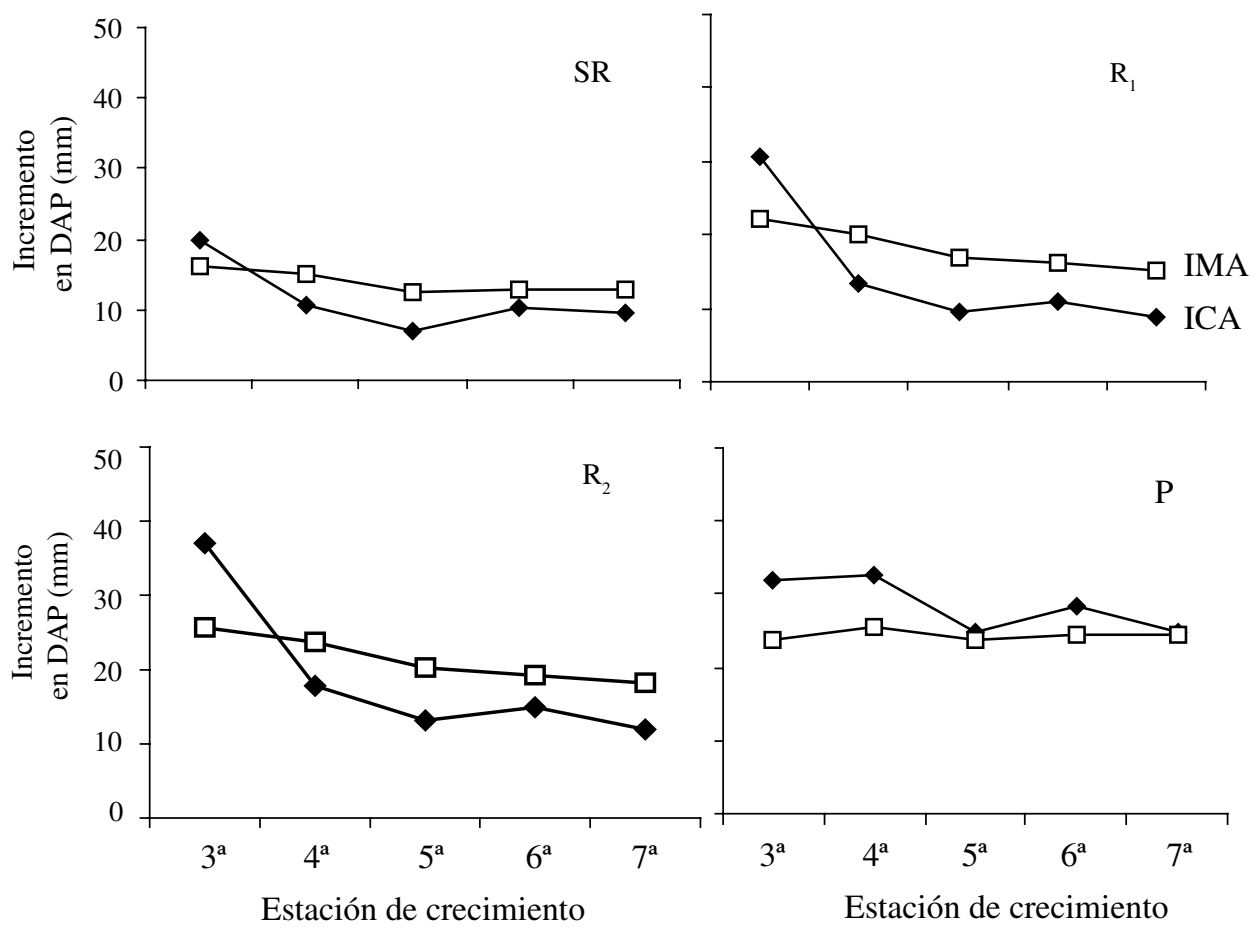

Figura 2. Evolución del incremento corriente anual (ICA) y del incremento medio anual (IMA) del DAP en plantas de Pinus elliottii, para cada tratamiento: regeneración natural sin raleo (SR), raleo dejando 5.000 árboles ha ${ }^{-1}\left(R_{1}\right)$, raleo dejando 2.500 árboles $h^{-1}\left(R_{2}\right)$ y plantación con 800 árboles $\mathrm{ha}^{-1}(\mathrm{P})$.

Evolution of the current annual increment (ICA) and the mean annual increment (IMA) of the breast height diameter (DAP) in Pinus elliottii seedlings for each treatment: natural regeneration without thinning (SR), thinning to residual density of 5,000 seedlings ha ${ }^{-1}\left(\mathrm{R}_{1}\right)$, thinning to residual density of 2,500 seedlings ha- ${ }^{-1}\left(\mathrm{R}_{2}\right)$, and planting with 800 seedlings ha ${ }^{-1}(\mathrm{P})$. 
Cuadro 2. Media ( \pm desviación estándar) de la altura total (A), el diámetro a la altura del pecho (DAP), relaciones alométricas y coeficiente de forma (CF) en plantas de Pinus elliottii, después de seis años de aplicados los tratamientos: regeneración natural sin raleo (SR), raleo dejando 5.000 árboles $h^{-1}\left(\mathrm{R}_{1}\right)$; raleo dejando 2.500 árboles ha ${ }^{-1}\left(\mathrm{R}_{2}\right)$ y plantación con 800 árboles ha ${ }^{-1}(\mathrm{P})$. Las letras diferentes en cada columna indican diferencias entre tratamientos (Tukey, $P<0,05$ ).

Mean ( \pm standard deviation) of total height (A), breast height diameter (DAP), allometric variables and cylindrical form factor (CF) in Pinus elliottii, six years after treatments were applied: natural regeneration without thinning (SR), thinning to residual density of 5,000 seedlings ha ${ }^{-1}$ $\left(\mathrm{R}_{1}\right)$, thinning to residual density of 2,500 seedlings ha ${ }^{-1}\left(\mathrm{R}_{2}\right)$, and planting with 800 seedlings ha ${ }^{-1}(\mathrm{P})$. Different letters in each column designate significant differences among treatments (Tukey, $P<0.05$ ).

\begin{tabular}{lrrcccc}
\hline & \multicolumn{1}{c}{ A $(\mathrm{m})$} & DAP $(\mathrm{cm})$ & DAP/DBT $(\mathrm{cm} / \mathrm{cm})$ & A/DAP $(\mathrm{m} / \mathrm{cm})$ & A/DBT $(\mathrm{m} / \mathrm{cm})$ & CF \\
\hline $\mathrm{SR}$ & $8,50 \pm 1,11 \mathrm{a}$ & $9,02 \pm 3,11 \mathrm{a}$ & $0,89 \pm 0,05 \mathrm{a}$ & $0,98 \pm 0,10 \mathrm{a}$ & $0,85 \pm 0,12 \mathrm{a}$ & $0,63 \pm 0,02 \mathrm{a}$ \\
$\mathrm{R}_{1}$ & $9,80 \pm 0,51 \mathrm{~b}$ & $10,51 \pm 1,62 \mathrm{~b}$ & $0,75 \pm 0,05 \mathrm{~b}$ & $0,89 \pm 0,10 \mathrm{~b}$ & $0,72 \pm 0,09 \mathrm{~b}$ & $0,55 \pm 0,02 \mathrm{~b}$ \\
$\mathrm{R}_{2}$ & $10,00 \pm 0,77 \mathrm{~b}$ & $12,75 \pm 1,11 \mathrm{c}$ & $0,77 \pm 0,01 \mathrm{~b}$ & $0,79 \pm 0,09 \mathrm{c}$ & $0,61 \pm 0,07 \mathrm{c}$ & $0,56 \pm 0,03 \mathrm{~b}$ \\
$\mathrm{P}$ & $9,50 \pm 0,53 \mathrm{~b}$ & $17,15 \pm 1,19 \mathrm{~d}$ & $0,72 \pm 0,09 \mathrm{c}$ & $0,59 \pm 0,06 \mathrm{~d}$ & $0,42 \pm 0,03 \mathrm{~d}$ & $0,53 \pm 0,02 \mathrm{c}$ \\
\hline
\end{tabular}

En la figura 2 se observa una caída del ICA durante la quinta estación de crecimiento, siendo este fenómeno más notable en la plantación. Esta caída estuvo asociada con la ocurrencia, durante ese año, de una sequía extraordinaria en la zona del estudio.

Seis años después de establecido el ensayo, el efecto de los diferentes tratamientos se reflejó en las características individuales de los árboles (cuadro 2). Se puede ver que el raleo tuvo un efecto significativo sobre el tamaño de los árboles, tanto en diámetro como en altura. Por otro lado, en la regeneración sin raleo los árboles fueron levemente más cilíndricos, mientras que en la plantación resultaron más cónicos. Esto se evidenció a través de los valores del CF y de las relaciones alométricas entre las distintas variables dimensionales. Esto muestra que el tratamiento sin raleo generó árboles morfológicamente muy delgados con relación a su altura, es decir, desbalanceados desde el punto de vista dimensional.

El análisis del crecimiento medio en altura de los 6 años de estudio, indicó que no hubo diferencias significativas entre la plantación $\left(1,22 \mathrm{~m}\right.$ año $\left.{ }^{-1}\right)$ y las poblaciones de regeneración natural raleadas $\left(R_{1}=1,23\right.$ y $R_{2}=1,28 \mathrm{~m}$ $\left.\mathrm{año} \mathrm{o}^{-1}\right)$. En cambio, la regeneración natural no raleada vio afectado su crecimiento medio en altura $\left(1,1 \mathrm{~m}\right.$ año $\left.{ }^{-1}\right)$, resultando su valor significativamente inferior $(P<0,05)$ respecto al resto de los tratamientos.

Competencia y crecimiento. Los valores de IDR fueron utilizados como indicadores del estado de competencia relativa entre las distintas poblaciones analizadas. Se pudo ver la existencia de un amplio rango de valores de IDR y un aumento del índice a través del tiempo (cuadro 3). Es necesario destacar que en el tratamiento SR se registró mortalidad de plantas a partir de la cuarta estación de crecimiento, y en $R_{1}$ este fenómeno ocurrió a partir de la quinta estación de crecimiento. En términos de IDR, podemos decir que este proceso se produjo cuando el índice superó el valor de 800. Pero la interpretación silvicultural de los valores obtenidos tiene sentido en la medida que los mismos puedan relacionarse con algún valor de IDR de refererencia y contrastarlos con datos de crecimiento. Este análisis será desarrollado en el capítulo de discusión.

Crecimiento y rendimiento. La integración entre el tamaño y la densidad de los árboles brinda una medida de la biomasa acumulada por unidad de superficie. En tal sentido, la misma fue cuantificada a través del área basal $(\mathrm{AB}) \mathrm{y}$ su evolución a través del tiempo (figura 3). Puede observarse que a medida que la densidad inicial es mayor el $\mathrm{AB}$ también aumenta, creciendo además a través del tiempo tal como lo hizo el IDR.

Luego de 6 años de iniciado el ensayo, el volumen total también se incrementó con el aumento de la densidad (cuadro 4). Pero la asociación entre el volumen total y el volumen comercial no fue lineal. En la regeneración natural sin raleo hubo más volumen pero sin valor comercial. En cambio, el raleo de la regeneración natural tuvo un efecto positivo sobre el rendimiento maderero, resultando diferente entre los tratamientos de acuerdo al tamaño comercial elegido.

Cuadro 3. Evolución del Índice de Densidad (IDR) en rodales de Pinus elliottii, para cada tratamiento: regeneración natural sin raleo (SR), raleo dejando 5.000 árboles $\mathrm{ha}^{-1}\left(\mathrm{R}_{1}\right)$, raleo dejando 2.500 árboles ha ${ }^{-1}\left(\mathrm{R}_{2}\right)$ y plantación con 800 árboles ha ${ }^{-1}(\mathrm{P})$.

Evolution of the Stand Density Index (IDR) in Pinus elliottii stands with different initial density for each treatment: natural regeneration without thinning (SR), thinning to residual density of 5,000 seedlings $\mathrm{ha}^{-1}\left(\mathrm{R}_{1}\right)$, thinning to residual density of 2,500 seedlings ha- ${ }^{-1}\left(\mathrm{R}_{2}\right)$, and planting with 800 seedlings ha ${ }^{-1}(\mathrm{P})$.

\begin{tabular}{lllrrr}
\hline \multicolumn{5}{c}{ Estación de crecimiento } \\
\cline { 2 - 6 } & \multicolumn{1}{c}{$3^{\mathrm{a}}$} & $4^{\mathrm{a}}$ & \multicolumn{1}{c}{$5^{\mathrm{a}}$} & \multicolumn{1}{c}{$6^{\mathrm{a}}$} & \multicolumn{1}{c}{$7^{\mathrm{a}}$} \\
\hline $\mathrm{SR}$ & 596 & 823 & 1.114 & 1.546 & 1.591 \\
$\mathrm{R}_{1}$ & 506 & 718 & 847 & 1.052 & 1.212 \\
$\mathrm{R}_{2}$ & 324 & 467 & 585 & 724 & 847 \\
$\mathrm{P}$ & 81 & 169 & 241 & 339 & 436 \\
\hline
\end{tabular}




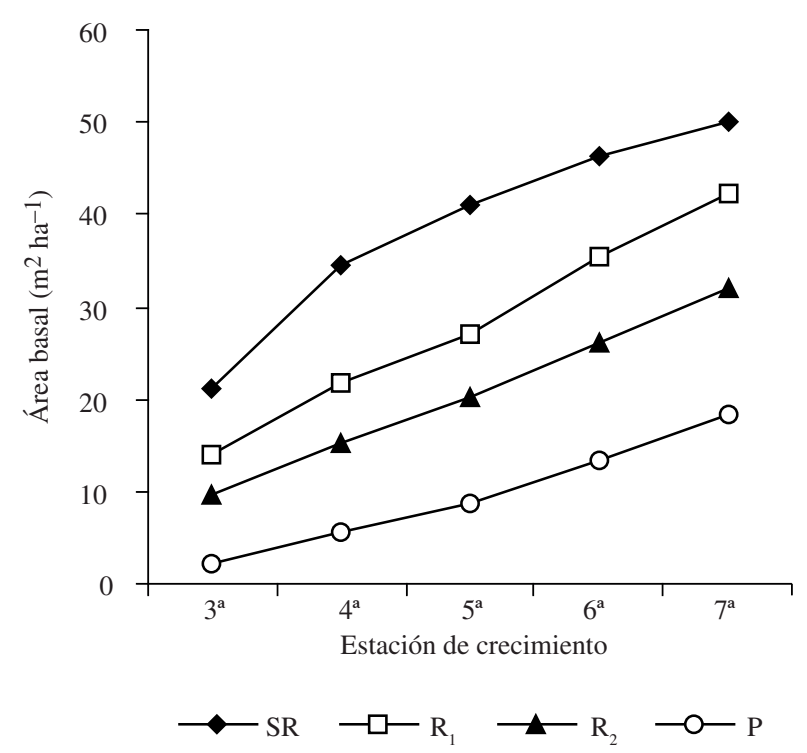

Figura 3. Evolución del área basal $\left(\mathrm{m}^{2} \mathrm{ha}^{-1}\right)$ en poblaciones de Pinus elliottii, para cada tratamiento: regeneración natural sin raleo (SR), raleo dejando 5.000 árboles ha ${ }^{-1}\left(\mathrm{R}_{1}\right)$, raleo dejando 2.500 árboles $h^{-1}\left(\mathrm{R}_{2}\right)$ y plantación con 800 árboles ha ${ }^{-1}(\mathrm{P})$.

Evolution of the basal area $\left(\mathrm{m}^{2} \mathrm{ha}^{-1}\right)$ in Pinus elliottii stands for each treatment: natural regeneration without thinning (SR); thinning to residual density of 5,000 seedlings $\mathrm{ha}^{-1}\left(\mathrm{R}_{1}\right)$, thinning to residual density of 2,500 seedlings ha-1 $\left(\mathrm{R}_{2}\right)$, and planting with 800 seedlings ha ${ }^{-1}(\mathrm{P})$.

Cuadro 4. Volumen total (VT) y volumen comercial de tallos con diámetro en punta fina $\geq 7 \mathrm{~cm}\left(\mathrm{VC}_{\geq 7}\right)$, y diámetro $\geq 10 \mathrm{~cm}$ $\left(\mathrm{VC}_{\geq 10}\right)$, en poblaciones de Pinus elliottii de siete años de edad, para cada tratamiento: regeneración natural sin raleo (SR), raleo dejando 5.000 árboles $\mathrm{ha}^{-1}\left(\mathrm{R}_{1}\right)$, raleo dejando 2.500 árboles ha $^{-1}\left(\mathrm{R}_{2}\right)$ y plantación con 800 árboles ha ${ }^{-1}(\mathrm{P})$. Largo de troza comercial $\geq 2 \mathrm{~m}$.

Total volume (VT) and commercial volume of stem with diameter $\geq 7 \mathrm{~cm}\left(\mathrm{VC}_{\geq 7}\right)$ and diameter $\geq 10 \mathrm{~cm}\left(\mathrm{VC}_{\geq 10}\right)$, in 7 year-old Pinus elliottii stands for each treatment: Natural regeneration without thinning (SR), thinning to residual density of 5,000 seedlings ha ${ }^{-1}\left(\mathrm{R}_{1}\right)$, thinning to residual density of 2,500 seedlings ha ${ }^{-1}\left(\mathrm{R}_{2}\right)$, and planting with 800 seedlings ha ${ }^{-1}(\mathrm{P})$. Length of commercial stem $\geq 2 \mathrm{~m}$.

\begin{tabular}{lccc}
\hline & $\mathrm{VT}\left(\mathrm{m}^{3} \mathrm{ha}^{-1}\right)$ & $\mathrm{VC}_{\geq 7}\left(\mathrm{~m}^{3} \mathrm{ha}^{-1}\right)$ & $\mathrm{VC}_{\geq 10}\left(\mathrm{~m}^{3} \mathrm{ha}^{-1}\right)$ \\
\hline $\mathrm{SR}$ & 372,34 & 34,05 & 0 \\
$\mathrm{R}_{1}$ & 227,44 & 168,85 & 110,27 \\
$\mathrm{R}_{2}$ & 174,28 & 162,60 & 142,21 \\
$\mathrm{P}$ & 92,34 & 90,20 & 84,50 \\
\hline
\end{tabular}

Tamaño de las ramas. La densidad de plantas también condicionó el tamaño de las ramas. La plantación (situación de menor densidad) tuvo ramas de mayor diámetro y longitud respecto a la regeneración natural (figura 4). La tendencia general fue una disminución significativa en el tamaño de las ramas a medida que la densidad inicial de plantas fue mayor.

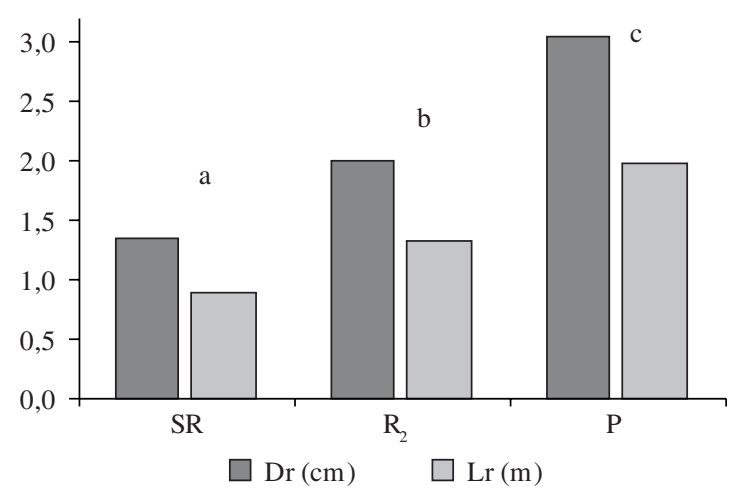

Figura 4. Diámetro (Dr) y longitud de ramas (Lr), en plantas de Pinus elliottii, después de seis años de aplicados los tratamientos: regeneración natural sin raleo (SR), raleo dejando 2.500 árboles $h^{-1}\left(\mathrm{R}_{2}\right)$ y plantación con 800 árboles ha ${ }^{-1}(\mathrm{P})$. Las letras diferentes indican diferencias entre tratamientos para las dos variables (Tukey, $P<0,05$ ).

Diameter (Dr) and length of the branches (Lr) in Pinus elliottii seedlings, six years after_density treatments were applied: natural regeneration without thinning (SR), thinning to residual density of 2,500 seedlings ha-1 $\left(\mathrm{R}_{2}\right)$, and planting with 800 seedlings ha ${ }^{-1}(\mathrm{P})$. Different letters designate significant differences among treatments in both variables (Tukey, $P<0.05$ ).

\section{DISCUSIÓN}

El menor tamaño de las plantas en $\mathrm{P}$ al inicio del estudio (cuadro 1) puede ser atribuido al denominado trauma de plantación (transplanting trauma), cuya causa principal es el estrés hídrico (South y Zwolinski 1997). Por el contrario, la regeneración natural no sufre de ese proceso, ya que cuenta con un sistema radical más desarrollado que permite acceder a fuentes de agua más profundas (Harrington et al. 1989). Resultados similares, respecto al mejor comportamiento inicial de la regeneración natural, fueron obtenidos en otros trabajos con coníferas (Harrington et al. 1989, South y Zwolinski 1997).

La mayor relación altura/diámetro en la regeneración natural respecto a la plantación es consecuencia de la temprana restricción en el crecimiento del diámetro respecto a la altura, debido a la alta densidad inicial. Los cambios en la relación altura/diámetro generalmente se asocian a una respuesta fisiológica de las plantas frente a situaciones de estrés, originado por competencia, por efecto del viento o por déficit hídrico.

Los resultados respecto al crecimiento en los primeros años (figura 1) ponen de manifiesto que el trauma de plantación es un proceso que puede extenderse más allá de las etapas iniciales. Esto coincide con lo reportado por Ackzell (1993), quien mostró que la plantación aventaja a la regeneración natural (tanto en diámetro como en altura), pero luego de algunos años.

Del análisis de la figura 2 surgen varios puntos que merecen ser desatacados. En primer lugar, se observa que al principio del ciclo el ICA es superior al IMA, y luego que 
ambas tasas se igualan se produce el fenómeno inverso (es decir, el IMA es superior al ICA). En segundo lugar, este proceso ocurre antes en el tratamiento con alta densidad (no raleado) respecto al resto. El estudio de Duryea (1987) también mostró una fuerte disminución en el crecimiento a partir del tercer año de edad de la regeneración natural, cuando la densidad superaba las 5.000 árboles $\mathrm{ha}^{-1}$. Otro aspecto que se evidencia en la figura 2 es que el valor en el cual el IMA y el ICA se igualaron fue inferior en el rodal sin raleo respecto al resto. Por lo tanto, el comportamiento de los incrementos (ICA e IMA) coincidió con el modelo general de evolución de las tasas de crecimiento a través del tiempo, aplicado tanto a nivel individual como poblacional (Bruce y Schumacher 1965, Husch et al. 1982). Finalmente, pudo verse que el efecto negativo de la sequía extraordinaria, ocurrida durante la quinta estación de crecimiento, afectó más a la plantación que a la regeneración natural. Esto supondría que, aún después de varios años, la regeneración natural mantiene su ventaja morfológica con relación al mejor desarrollo del sistema radical.

En términos generales, los resultados del presente estudio indican que el raleo temprano de la regeneración tiene un efecto positivo sobre el crecimiento individual. Similares resultados se encontraron en Pinus taeda L (una especie afín a $P$. elliottii), cuando se realizó un raleo químico de la regeneración natural (Keister y McGriff 1974). En la misma zona del presente trabajo, Silva (2000) reportó un valor de IMA para el diámetro de $1,9 \mathrm{~cm}$ año-1, en una regeneracion natural de 10 años que había sido raleada a los cuatro años de edad, dejando 850 árboles $\mathrm{ha}^{-1}$.

La depresión del crecimiento en altura ocurrida en SR (cuadro 2) no es un fenómeno común en un amplio rango de densidades, dentro del cual la altura es una variable densidad-independiente (Daniel et al. 1982). Por lo tanto, se puede concluir que la regeneración no raleada alcanzó niveles muy elevados de competencia que afectaron el crecimiento en altura.

Los resultados mostraron además que el crecimiento de la regeneración raleada fue muy bueno, e incluso superior al registrado en plantaciones de la misma edad en el área de origen de la especie (Dalla Tea y Jokela, 1991). En un estudio realizado en la provincia de Corrientes (al norte de la zona del presente estudio), plantaciones de P. elliottii de cuatro años de edad alcanzan 8 a $11 \mathrm{~cm}$ de DAP, y cinco metros de altura (Pezzutti y Caldato 2004). Por lo tanto, se puede decir que las poblaciones que fueron evaluadas en el presente estudio presentaron un alto potencial de crecimiento.

La interpretación silvicultural de los valores de IDR obtenidos se puede hacer a partir de los estudios de Long (1985), y de Dean y Baldwin (1993), quienes trabajaron con varias coníferas (entre ellas P. taeda). Estos autores proponen manejar la densidad dentro del rango comprendido entre el 35\% y el $60 \%$ del máximo IDR de cada especie, el cual corresponde al rango de máxima productividad (volumen $\mathrm{ha}^{-1} \mathrm{año}^{-1}$ ) que fuera definida por Langsaeter
(1941). El límite inferior (35\%) está dado por la densidad debajo de la cual habría una subutilización del espacio de crecimiento, y corresponde aproximadamente al momento del cierre de las copas. El límite superior (60\%) queda definido por la denominada línea de inminente mortalidad por competencia, por encima de la cual las poblaciones entran en un proceso de autorraleo. Dentro de las opciones de manejo también existe la posibilidad de llevar a los rodales hasta un $25 \%$ del IDR máximo, lo cual implica una menor tasa de crecimiento por unidad de superficie. Sin embargo, esta alternativa puede adoptarse siempre y cuando exista un crecimiento individual en diámetro que lo justifique económicamente.

El valor más elevado de IDR que hallamos en bibliografía para P. elliottii fue de 1.450 (Borzone y Bardi 1992), y en el presente trabajo fue cercano a 1.600. En el trabajo de Rebottaro et al. (2003) se muestra que las plantaciones de adultos de $P$. elliottii sometidos a un régimen de raleo presentaron valores de IDR entre 600 y 800 . En cambio, los rodales con un valor de IDR mayor a 900 se encontraban en proceso de autorraleo. En el presente estudio, la mortalidad de plantas se registró cuando los rodales sobrepasaron un IDR de 800. Tomando como base toda la información anterior, se puede inferir que en términos de IDR, la regeneración natural sin raleo presentó tempranamente muy alta competencia (cuadro 3), coincidiendo con las bajas tasas de crecimiento diamétrico (figura 2), similar a lo reportado por Duryea (1987). Por otro lado, la población con un raleo menos intenso (5.000 árboles $\mathrm{ha}^{-1}$ ) presentó un alto nivel de competencia a los tres años del tratamiento, lo cual fue coherente con la depresión en el crecimiento individual. En cambio, la plantación, al tener bajos niveles de densidad, presenta valores altos de crecimiento individual.

La descripción de la acumulación de biomasa con el aumento de la densidad inicial, concuerda con los modelos de crecimiento poblacional en sistemas forestales (Piennar y Turnbull 1973). Así, luego de seis años de establecido el ensayo, la regeneración natural sin raleo alcanzó mayor $\mathrm{AB}$ y volumen, aunque el mismo carece de valor comercial debido a que los árboles son pequeños.

Asumiendo un IDR máximo en $P$. elliottii de alrededor de 1.500 , se sugiere que las poblaciones se manejen entre valores de 500 y 800 de IDR. Sin embargo, el rango finalmente adoptado dependerá del objetivo de producción. Por ejemplo, si se pretende obtener mucho volumen en poco tiempo, a expensas de menores tasas de crecimiento individual, nos ubicamos en los rangos de IDR más altos (por ej. 650-800). En principio, la población sometida a un raleo menos intenso (5.000 árboles ha ${ }^{-1}$ ) puede responder a este planteamiento. Pero para mantener el rodal productivo es necesario realizar un segundo raleo alrededor de los 5 o 6 años, a fin de no comprometer fuertemente el crecimiento futuro y la sobrevivencia de las plantas.

$\mathrm{Si}$ el objetivo es obtener madera de calidad, es decir, diámetros grandes en el menor tiempo posible, se debería 
ubicar en los valores menores del rango (por ej. 500-650 de IDR). La población raleada más intensamente $(2.500$ árboles $\mathrm{ha}^{-1}$ ) podría responder a este esquema productivo, sometiéndola a un primer raleo de tipo comercial alrededor de los 6-7 años de edad.

Moorhead et al. (1997) recomiendan efectuar un primer raleo entre los 3 y 5 años de edad, pudiendo utilizar en esta etapa un tratamiento mecánico a un costo relativamente bajo. En la zona donde se llevó a cabo el presente trabajo, Silva (2000) estimó que el raleo precomercial de la regeneración tiene un costo aproximado de 3 a 4 jornales $\mathrm{ha}^{-1}$. Considerando, además, que en Argentina la ley de inversiones forestales contempla la posibilidad de subsidiar el primer raleo, un sistema de producción que utiliza la regeneración natural puede resultar económicamente atractivo, aunque haya que efectuar un control inicial de la densidad.

Tanto en $R_{1}$ como en $R_{2}$ el segundo raleo a los siete años permite obtener entre 70 y $80 \mathrm{~m}^{3} \mathrm{ha}^{-1}$, con destino a la industria de la trituración. Este es un mercado disponible en la región, ya que existe una empresa de tableros que consume materia prima de diámetros pequeños.

Finalmente, la diferencia observada en el tamaño de las ramas (figura 4) estaría asociada a la historia de competencia de los individuos para cada población analizada. En la plantación, los árboles transcurrieron la mayor parte del tiempo bajo crecimiento libre, lo cual permitió el desarrollo de ramas más gruesas y largas. En la regeneración no raleada, la elevada densidad restringió muy temprano el desarrollo de la copa. El raleo aumentó el espacio potencial de crecimiento individual, permitiendo que el mismo se refleje en el desarrollo de copa, a través de la dimensión de sus ramas. La implicancia de este resultado, desde el punto de vista de manejo, se presenta en caso que el objetivo productivo sea obtener algunas trozas de madera libre de nudos en la cosecha final. En este caso, la primer poda puede resultar más económica en la regeneración natural que en la plantación. Las poblaciones como $R_{2}$ podrían ajustarse a este objetivo, para lo cual después de efectuar el segundo raleo (o primer raleo comercial) puede iniciarse un programa de podas en los árboles remanentes.

\section{CONCLUSIONES}

En poblaciones densas de $P$. elliottii provenientes de regeneración natural, el raleo temprano tiene un efecto positivo sobre el crecimiento en diámetro y altura.

La regeneración natural raleada presenta un potencial de crecimiento individual similar al registrado en plantaciones comerciales, donde se utiliza material genético mejorado.

El manejo de la densidad en $P$. elliottii debería efectuarse manteniendo a los rodales dentro de valores de IDR entre 500 y 800 , dependiendo del objetivo productivo.
Luego de seis años de evaluación, la regeneración natural sin raleo acumula mucho volumen por ha, pero sin valor comercial.

Las dos intensidades de raleo precomercial aplicadas (5.000 y 2.500 árboles ha $^{-1}$ ) permiten obtener un alto volumen de madera comercial de pequeñas dimensiones, en pocos años. En cambio, la regeneración sometida a un raleo más intenso (2.500 árboles $\mathrm{ha}^{-1}$ ) es el tratamiento que alcanza un mayor volumen comercial de dimensiones más grandes.

El diámetro y la longitud de ramas de los árboles disminuyó con el aumento de la densidad de plantas.

Los resultados mostraron que la regeneración natural manejada representa una alternativa productiva frente a la plantación tradicional.

\section{AGRADECIMIENTOS}

Los autores quieren agradecer a los Ingenieros Martín Marcó, Diana Díaz, Martín Sánchez Acosta, Graciela Rembado y Federico Larocca del Instituto Nacional de Tecnología Agropecuaria (INTA). También agradecen a los estudiantes Santiago Cordeiro y Rubén Cerrillo, por su colaboración en el trabajo de campo. El estudio fue financiado por los proyectos UBACyT G005 (Secretaría de Ciencia y Técnica de la Universidad de Buenos Aires) y los Proyectos de Investigación Aplicada PIA 14/96 y 34/98, Proyecto Forestal de Desarrollo, Secretaría de Agricultura, Ganadería, Pesca y Alimentación, Argentina.

\section{REFERENCIAS}

Ackzell L. 1993. A comparison of planting, sowing, and natural regeneration for Pinus sylvestris (L.) in boreal Sweden. Forest Ecology and Management 61(3-4): 229-245.

Backer J, P Muphy. 1982. Growth and yield following four reproduction cutting. Methods in loblolly-shortleaf pine. A case study. Southern Journal of Applied Forestry 6(2): 66-74.

Barnett JP, JM McGilvray. 1991. Carry-over of loblolly pine seeds on cutover forest sites. Tree Planters' Notes 42(4): 17-18.

Borzone HA, JF Bardi. 1992. Determinación del crecimiento de una parcela de Pinus elliottii de 10 años en Azul (Buenos Aires). En Actas Jornadas sobre pinos subtropicales. Buenos Aires, Argentina. Tomo I, p. 180-188.

Bruce D, F Schumacher. 1965. Medición Forestal. Primera edición en español. Trad. Palazon, R. y Meza Nieto, J. Editorial Herrero. México. 474 p.

Brassiolo M, R Seitz. 1988. Regeneración natural de Pinus elliottii Engelm, bajo cobertura. In Actas VI Congreso Forestal Argentino. Santiago del Estero, Argentina. Tomo II, p. 357-359.

Cabrelli D, S Rebottaro, C Winckler. 1997. Dinámica de poblaciones jóvenes de regeneración natural de Pinus elliottii Engelm. en el subtrópico húmedo de Argentina. En: XI Congreso Forestal Mundial. Antalya, Turquía. Vol. 3, p. 65. 
Cain MD. 1987. Site-Preparation techniques for establishing natural pine regeneration on small forest properties. Southern Journal of Applied Forestry 11(1): 41-45.

Cain MD. 1991. Importance of seedyear, seedbed, and overstory for establishment of natural loblolly and shortleaf pine regeneration in southern Arkansas. Forest Service Research Paper SO-268. USDA. 10 p.

Cain MD. 1996. Growth expectations from alternative thinning regimes and prescribed burning in naturally regenerated loblolly-shortleaf pine stands through age 20. Forest Ecology and Management 81(1-3): 227-241.

Cain MD, MG Shelton. 2001. Twenty years of natural loblolly and shortleaf pine seed production on the Crossett Experimental Forest in southeastern Arkansas. Southern Journal of Applied Forestry 25(1): 40-45.

Cozzo D, D Cabrelli, S Cozzo, J Darraidou, M Dimitri, J Gambini, C Rezzano, M R Larrieu, A Torres. 1988. Introducción a una propuesta de plantaciones asilvestradas como alternativa de transición entre la economía maderera y la conservación ambiental. Primeros resultados obtenidos en la Argentina en Pinus elliottii y Pinus taeda. Ed. Facultad de Agronomía, Universidad de Buenos Aires. $71 \mathrm{p}$.

Dalla Tea F, EJ Jokela. 1991. Needlefall, canopy light, interception, and productivity of young intensively managed slash and loblolly pine stands. Forest Science 37(5): 1298-1313.

Dangerfield CWJr, MB Edwards. 1991. Economic comparison of natural and planted regeneration of loblolly pine. Southern Journal of Applied Forestry 15(3): 125-127.

Daniel PW, U Helms, FS Baker. 1982. Principios de Silvicultura. Ed. McGraw-Hill. 492 p.

De Bellis S. 1986. Contribución para el estudio sobre la regeneración natural de Pinus elliottii-taeda en el Delta inferior del río Paraná. Tesis Ingeniero Agrónomo. Buenos Aires, Argentina. Facultad de Agronomía, Universidad de Buenos Aires. 38 p.

Dean TJ, VC Baldwin. 1993. Using a Density-Management Diagram to Develop Thinning Schedules for loblolly pine plantations. Forest Service Research Paper SO-275. USDA. 7 p.

Duryea ML. 1987. Forest Regeneration Methods: natural regeneration, direct seeding and planting. Cir. 759. Cooperative Extension Service, University of Florida, Institute of Food and Agriculture Science. $10 \mathrm{p}$.

Edwards MB. 1987. Natural Regeneration of Loblolly Pine: A Loblolly Pine Management Guide. USDA. Forest Service General Technical Report SE-47. USDA 17 p.

Harrington A, C Brissette, C Carlson. 1989. Root system structure in planted and seeded loblolly and shortleaf pine. Forest Science 35(2): 469-480.

Husch B, CI Miller, TW Beers. 1982. Forest Mensuration. Third Edition. John Wiley \& Sons. USA. 402 p.

Keister TD, JA McGriff. 1974. Thinning dense young loblolly pine stands with a granular herbicide. Forestry Notes $\mathrm{N}^{\circ}$ 107. Louisiana State University. $4 \mathrm{p}$.

Langdon OG. 1981. Some effects of prescribed fire on understory vegetation in loblolly pine stands. In Wood GW (Ed.). Prescribed fire and wildfire in southern forests: symposium proceedings. Belle W. Baruch Forest Science Institute, Georgetown, South Carolina. p. 143-153.

Langdon OG, F Bennett. 1976. Management of natural stands of slash pine. Forest Service Research Paper SE-147. USDA. 12 p.
Langsaeter A. 1941. Om tynning i enaldret gran-og furuskog (About thinning in even-aged stands of spruce, fir and pine), Meddelf.d. Norske Skogforsoksvesen 8:131-216.

Lohrey RE, SV Kossuth. 1990. Pinus elliottii Engelm. slash pine. In: Burns, Russell M; Honkala, Barbara H, technical coordinators. Silvics of North America. Vol. 1. Conifers. Agric. Handb. 654. Washington, DC: USDA, Forest Service. p. 338-347.

Lombardi C. 1982. Índice de crecimiento de especies en la provincia de Corrientes. Revista Asociación Forestal Argentina 26: 19-31.

Long JN. 1985. A practical approach to Density Management. Forestry Chronicle 61(1): 23-27.

Mann WFJr, RE Lohrey. 1974. Precommercial thinning of southern pines. Journal of Forestry 72: 557-560.

McCulley, RD. 1950. Management of natural slash pine stands in the Flatwoods of south Florida and north Florida. Circular $\mathrm{N}^{\circ}$ 845. USDA. 57 p.

Méndez H. 1996. Estudio de la estructura y composición de la hojarasca y su relación con el proceso de regeneración natural en plantaciones de $P$. elliottii Engelm. y P. taeda Lin., en la Mesopotamia Argentina. Tesis Ingeniero Agrónomo. Buenos Aires, Argentina. Facultad de Agronomía, Universidad de Buenos Aires. 29 p.

Moorhead DJ, CWJr Dangerfield, MB Edwards. 1997. Regulating Stand Density by Precommercial Thinning in Naturally Regenerated Loblolly Pine Stands: Evaluation of Management and Economic Opportunities. In Proceedings of the Ninth Biennial Southern Silvilcultural Research Conference, Clemson, SC, Feb. 25-27, 1997. USDA General Technical Report SRS-20.

Pezzutti RV, SL Caldato. 2004. Efecto del control de malezas en el crecimiento de plantaciones de Pinus taeda, Pinus elliottii var. elliottii y Pinus elliottii var. elliottii $x$ Pinus caribaea var. hondurensis. Bosque 25(2): 77-87.

Piennar LV, KJ Turnbull. 1973. The Chapman-Richards generalization of Von Bertalanffy's growth model for basal area growth and yield in even-aged stands. Forest Science 19(4): 2-22.

Rebottaro S, D Cabrelli, C Winckler, C Rezzano, A Torres. 2001. Precommercial thinning of slash pine natural regeneration, in Argentina. In $11^{\text {th }}$ Biennial Southern Silvicultural Research Conference. Knoxville, TN, March 2001, USA. p. 44.

Rebottaro S, D Cabrelli, P Rodríguez. 2002. Effects of establishment method (artificial and natural regeneration) on the biomass accumulation in Pinus elliottii Engelm. var. elliottii stands. In Popular Summaries Fourth International Conference on Forest Vegetation Management. Ed. H. Frochot, C. Collet, P. Balandier. INRA-CEMAGREF, France. 387-389.

Rebottaro S, D Cabrelli, E Rivero, D Ceballos, E Rienzi. 2003. Importancia del mantillo forestal de pinos subtropicales en suelos de baja fertilidad. Commission B4: Management and development of forests land. XII Congreso Forestal Mundial. Quebec, Canadá. Consultado: 14/08/04. Disponible en: www. fao.org/DOCREP/ARTICLE/WFC/XII/0369-B4.htm

Rebottaro S, D Cabrelli. 2004. Efecto del sistema de establecimiento (plantación y regeneración natural) de pinos subtropicales sobre las propiedades del suelo y la disponibilidad de nutrientes. En: Secretaría de Agricultura, Ganadería, Pesca y Alimentación. En Investigación Forestal al Servicio de la Producción. Segunda Entrega. Ed. Proyecto Forestal de Desarrollo. Buenos Aires, Argentina. p. 123-127. 
Rebottaro S, D Cabrelli, A Gowland. 2004. Reclutamiento, mortandad y crecimiento de la regeneración natural de Pinus elliottii establecida en aperturas del bosque con diferente geometría. III Simposio Internacional sobre Manejo Sustentable de Recursos Forestales. Pinar del Río, Cuba. Disponible en CD (ISBN 959-16-0261-X).

Reineke LH. 1933. Perfecting a stand-density index for even-aged forests. Journal Agricultural Research 46(7): 627-638.

SAS Institute Inc. 2001. SAS User guide: statistics. SAS Institute Inc., Cary, NC.

SAGyP (Secretaría de Agricultura, Ganadería y Pesca, AR) 1999. Argentina: oportunidades de inversión en bosques cultivados. Buenos Aires, Argentina. 208 p.
SMN (Servicio Meteorológico Nacional). 1992. Datos climáticos 1961-1990. EEA INTA Concordia, SMN, Fuerza Aérea, Argentina.

Silva D. 2000. Regeneración natural de Pinus elliottii de 10 años de edad. En XV Jornadas Forestales de Entre Ríos. INTA, Concordia, Entre Ríos, Argentina. Manual de campo, p. 7-8.

South DB, JB Zwolinski. 1997. Transplant stress index: a proposed method of quantifying planting check. New Forests 13(1): 315-328.

Torres A, D Cabrelli, C Winckler, S Rebottaro, C Rezzano. 1994. Evaluación preliminar de la regeneración natural de Pinus elliottii Engelm. en Gualeguaychú, provincia de Entre Ríos. Revista de Facultad Agronomía 14(3): 219-227.

Recibido: 19.04 .06

Aceptado: 06.06.07 